INPLASY

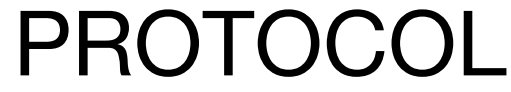

To cite: Li et al. Moxibustion for abdominal pain in COVID-19A protocol for systematic review and metaanalysis. Inplasy protocol 2021120104. doi:

10.37766/inplasy2021.12.0104

Received: 23 December 2021

Published: 23 December 2021

Corresponding author:

Xuhao Li

so_nn@qq.com

Author Affiliation:

Shandong University of traditional Chinese Medicine.

Support: None.

Review Stage at time of this submission: The review has not yet started.

Conflicts of interest:

None declared.

\section{Moxibustion for abdominal pain in COVID-19 A protocol for systematic review and meta-analysis}

Li, XH¹; Dong, TT2; Hou, Y3; Dong, ZB4; Yang, JG5.

Review question / Objective: How do moxibustion treat COVID-19 abdominal pain?

Condition being studied: A systematic evaluation of the effect of moxibustion on COVID-19 abdominal pain.

Information sources: The Cochrane Central of Controlled Trials (CENTRAL), PubMed, Embase, Chinese Biomedical Literature Database, CNKI, Weipu Chinese Science and Technology Journal Database, Wanfang Database, and related journals will be searched. The search time will be from the establishment of the database-2021.12.31. Randomized clinical trials and retrospective cohort studies will be included.

INPLASY registration number: This protocol was registered with the International Platform of Registered Systematic Review and Meta-Analysis Protocols (INPLASY) on 23 December 2021 and was last updated on 23 December 2021 (registration number INPLASY2021120104).

\section{INTRODUCTION}

Review question / Objective: How do moxibustion treat COVID-19 abdominal pain?

Condition being studied: A systematic evaluation of the effect of moxibustion on COVID-19 abdominal pain.

\section{METHODS}

Participant or population: Patients with COVID-19 abdominal pain.

Intervention: The intervention measures in the experimental group should contain moxibustion. 
Comparator: As included in eligible randomized clinical trials and retrospective cohort studies.

Study designs to be included: Randomized clinical trials and retrospective cohort studies will be included.

Eligibility criteria: Subjects with documented COVID-19 with abdominal pain lasting two weeks or longer. There were no restrictions on sex, education, race, or disease stage, aged between 18 and 65 years. Patients with a history of abdominal pain prior to COVID-19 infection were excluded.

Information sources: The Cochrane Central of Controlled Trials (CENTRAL), PubMed, Embase, Chinese Biomedical Literature Database, CNKI, Weipu Chinese Science and Technology Journal Database, Wanfang Database, and related journals will be searched. The search time will be from the establishment of the database-2021.12.31. Randomized clinical trials and retrospective cohort studies will be included.

Main outcome(s): Effectiveness indicators.Clinical variables will be set as the effectiveness indicators, such as the abdominal pain frequency, abdominal pain intensity, duration of abdominal pain, times of using pain killer, and quality of life. The abdominal pain frequency, abdominal pain intensity are dichotomous variables. The dichotomous outcomes will be described by relative risk (RR) and $95 \%$ confidence interval $(\mathrm{Cl})$, in addition, mean difference (MD) and $95 \% \mathrm{Cl}$ will be used to describe the effect value of the inter-group comparison.

Additional outcome(s): The adverse reactions are continuous variables.

Quality assessment / Risk of bias analysis: The methodological quality of each included study will be assessed independently by two reviewers using two tools. The Cochrane collaboration tool will be used to assess the quality of randomized controlled trials. It comprises the following 7 aspects: random sequence generation, allocation concealment, blind method, incomplete result data, selective reporting, and other biases. The quality assessment results of each item can be divided into three grades: "low risk", "high risk" and "unclear". The more rigorous the design and the higher the methodological quality of each RCT, the lower the risk coefficient. The Newcastle Ottawa Scale (NOS) will be used to assess the quality of retrospective studies. This method includes three aspects to the evaluation: the selection method, comparability and contact exposure assessment method of case group and control group. The higher the score, the higher the quality of the study. When necessary, the consensus on this issue will be studied with the help of a third party.

Strategy of data synthesis: The RevMan5.3 software provided by the Cochrane website will be used for the analysis. Categorical variables will be expressed by odds ratio (OR) and marked with $95 \%$ confidence interval $(\mathrm{Cl})$. Continuous variables will be expressed by mean difference (MD) and marked with $95 \% \mathrm{Cl}$. If $\mathrm{P} 0.1$ or $\mathrm{I}^{2}<50 \%$, it means that the heterogeneity between groups is small, and the fixed effect model will, in this case, be used for combined analysis; when $\mathrm{P} 50 \%$, it shows that the heterogeneity between the groups is large, and the random effects model will then be used for combined analysis, and the results shown in forest plots. Analysis of potential publication bias will be shown in a funnel chart, and sensitivity analysis and subgroup analysis will be used when necessary.

Subgroup analysis: Subgroup analysis will be used to evaluate the therapeutic effects among different drugs. Inverted funnel plots and Egger's regression test will be used to determine publication bias when the number of included studies exceeds 10 in the network meta-analysis.

Sensitivity analysis: We carried out a sensitivity analysis to investigate the robustness of the conclusions. The principal decision nodes include the 
method quality, sample size, and impact of missing data. Therefore, the impact of lowquality research on overall results was assessed.

Country(ies) involved: China.

Keywords: moxibustion, abdominal pain, protocol, coronavirus disease 2019.

Contributions of each author:

Author 1 - Xuhao LI.

Email: 563500460@qq.com

Author 2 - Tiantian Dong.

Email: sddongtt@163.com

Author 3 - Yi Hou.

Email: 937157330@qq.com

Author 4 - Zhibin Dong.

Email: dzb2019zj@126.com

Author 5 - Jiguo Yang.

Email: so_nn@qq.com 